\title{
Age, Obesity, Family History, Previous Gestational Diabetes Are Major Risk Factors for Hyperglycemia in Pregnant Bulgarian Women
}

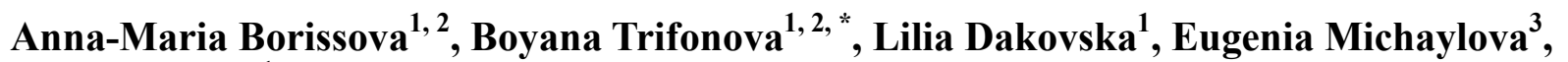 \\ Mircho Vukov ${ }^{1}$ \\ ${ }^{1}$ Clinic of Endocrinology, University Hospital Sofiamed, Sofia, Bulgaria \\ ${ }^{2}$ Faculty of Medicine, Sofia University Saint Kliment Ohridski, Sofia, Bulgaria \\ ${ }^{3}$ Medical Diagnostic Laboratory Bodimed, Sofia, Bulgaria
}

Email address:

boianatri@abv.bg (B. Trifonova)

${ }^{*}$ Corresponding author

\section{To cite this article:}

Anna-Maria Borissova, Boyana Trifonova, Lilia Dakovska, Eugenia Michaylova, Mircho Vukov. Age, Obesity, Family History, Previous Gestational Diabetes Are Major Risk Factors for Hyperglycemia in Pregnant Bulgarian Women. European Journal of Preventive Medicine. Vol. 9, No. 2, 2021, pp. 39-45. doi: 10.11648/j.ejpm.20210902.12

Received: February 20, 2021; Accepted: March 8, 2021; Published: March 22, 2021

\begin{abstract}
The frequency of Gestational Diabetes Mellitus (GDM) ranges from 5.2\% to $40.4 \%$ in different countries. This wide variability is due to numerous risk factors. The aim of the present study was to analyze the frequency and role of some risk factors for the development of Hyperglycemia regarding the Bulgarian population of pregnant women. Material: We screened 547 pregnant women, mean age $30.49 \pm 5.12$ years, divided into two groups: up to 24 gestational week - g.w. (n-386, 70.6\%) and after 24 g.w. (n-161, 29.4\%). Methods: BMI before pregnancy and the current one at the time of the study were calculated. A two-hour, $75 \mathrm{~g}$ oral glucose tolerance test (OGTT) was performed. Plasma glucose was quantitatively determined using enzymatic reference method with hexokinase (Roche reagent) on Cobas e501 analyzer, in one Central laboratory on the day of the blood sampling. The results were in $\mathrm{mmol} / 1$. The statistical analysis was performed using standard SPSS 13.0 for Windows. Results: We showed that $14.4 \%$ (n-79/547) of the screened pregnant women have Hyperglycemia in fasted state or during the OGTT test $-7.5 \%$ in group up to 24 g.w. versus $31 \%$ in group after 24 g.w, $\mathrm{P}<0.01$. The age of the group of pregnant women with Hyperglycemia (n-79) versus the others with Normoglycemia (n-468) was significantly higher $-32.18 \pm 5.26$ years v.s. $30.21 \pm 5.05$ years, $\mathrm{P}<0.005$. The women with a higher BMI before and during pregnancy are significantly at risk of developing glucose tolerance disorders during pregnancy, $\mathrm{P}<0.0001$. Family history of diabetes occurred in $29.1 \%(\mathrm{n}-23 / 79)$ of the pregnant women with Hyperglycemia, versus $13.5 \%$ (n-63/468) of those with Normoglycemia, $\mathrm{P}<0.001$. There was a significant difference between the incidence of Hyperglycemia in pregnant women with previous GDM compared with those without - 3/79 $(3.8 \%)$ versus $3 / 468(0.6 \%), \mathrm{P}<0.04$. Conclusion: Considering the main significant risk factors for the development of Hyperglycemia during pregnancy, identified in our population in the current screening - advanced maternal age, obesity, family history of diabetes, previous GDM or High blood sugar before pregnancy, verbal screening would be very helpful and would direct us immediately to screening each pregnant woman with any of these risk factors.
\end{abstract}

Keywords: Pregnancy, Hyperglycemia, Maternal Age, Obesity, Family History of Diabetes, Previous Gestational Diabetes

\section{Introduction}

The International Diabetes Federation (IDF) estimates in Diabetes Atlas (2019) that 223 million women worldwide (20-79 years) live with diabetes and 20 million or $16 \%$ of successful births occur in conditions of hyperglycemia during pregnancy. The Hyperglycemia is one of the most common conditions during pregnancy. Most cases of Hyperglycemia during pregnancy are in countries with low and medium gross domestic product, where access to health care for women is limited [1]. Since 1965, the World Health Organization (WHO) 
has been working on the classification of types of diabetes mellitus, one of which is diabetes during pregnancy [2, 3]. In 2014, special WHO'2014 Recommendations were published on the classification of Hyperglycemia in pregnancy [4].

The age of onset of type 2 diabetes mellitus (T2D) is declining worldwide, and many women with previously unknown T2D can become pregnant, and their diabetes can be diagnosed by routine tests during pregnancy [1]. In addition, women at high risk of diabetes cannot withstand metabolic stress during pregnancy and may develop diabetes for the first time in this period of their lives.

In cases of newly diagnosed diabetes (existing but unknown before pregnancy), the oral glucose tolerance test (OGTT), conducted in the first half of pregnancy (12-16 weeks) according to the generally accepted population criteria for diabetes in non-pregnant women, confirms this: fasting plasma glucose $(\mathrm{FPG}) \geq 7.0 \mathrm{mmol} / \mathrm{L}$ and / or at 2 hours $\geq 11.1$ $\mathrm{mmol} / \mathrm{L}$ or random plasma glucose $\geq 11.1 \mathrm{mmol} / \mathrm{L}$. When the level of Hyperglycemia, first detected by testing any time during the course of pregnancy, meets the criteria for diagnosis of diabetes in the nonpregnant state, the condition is called Diabetes in Pregnancy (DIP) [5, 6].

Another type of Hyperglycemia is when it is detected during pregnancy (usually between 24 and 28 weeks of gestation) and is assessed according specific criteria for diagnosing Gestational Diabetes Mellitus (GDM) adopted by NICE: plasma fasting glycemia $\geq 5.6 \mathrm{mmol} / \mathrm{L}$ or plasma glycemia at 120-th minute of OGTT $\geq 7.8 \mathrm{mmol} / \mathrm{L} \mathrm{[7].}$

The International Federation of Gynecology and Obstetrics - FIGO (2015) and WHO (2019) use slightly different criteria for GDM - hyperglycemia above the diagnostic threshold for gestational diabetes: fasting plasma glucose $5.1-6.9 \mathrm{mmol} / \mathrm{L}$, plasma glucose at 1 hour $\geq 10.0 \mathrm{mmol} / \mathrm{L}$, plasma glucose at 2 hours 8.5 - $11.0 \mathrm{mmol} / \mathrm{L}[5,6]$.

According to the IDF, the prevalence of GDM is in the wide range of $1-28 \%[8,6]$. Other authors find that the frequency of GDM ranges from $5.2 \%$ to $40.4 \%$ in different countries. This wide variability reflects numerous risk factors, including the size of Gross Domestic Product per capita [9]. Based on the criteria of the Association of Diabetes in Pregnancy Study Group (IADPSG), the European incidence of GDM is estimated to be around $13 \%$ [10].

In our study of this issue 547 pregnant Bulgarian women were examined in detail and the frequency and characteristics of glucose tolerance disorders during pregnancy were analyzed, according to modern criteria $[5,6,7]$. We found Hyperglycemia in $14.4 \%$ (79/547) of pregnant Bulgarian women. Its frequency increased with the progress of pregnancy and almost doubled after 24 gestational week (g. w.). Only $8.9 \%(7 / 79)$ of the pregnant women were newly diagnosed with Diabetes mellitus, according to the criteria for the general population, ie. Diabetes in Pregnancy (DIP), while the remaining cases met the criteria for GDM - 91.1\% [11].

In diabetics, the intrauterine environment can lead to placental dysfunction and hormonal disorders, which can affect the mother, the course of pregnancy, the birth process as well as the fetus $[12,13]$.
It should be noted that the frequency of GDM is increasing globally. There is an increase in the age of pregnancy of women, which is a worldwide trend having various social, financial and personal reasons. Analyzes show that the incidence of Hyperglycemia during pregnancy increases with the age of the pregnant woman, reaching its highest point after the age of 45 [1]. In addition to the mother's advanced age, there are some other risk factors for GDM such as obesity / overweight, previous GDM or accidentally elevated blood sugar and family history of diabetes [14]. A family history of diabetes is present in case of at least one first-degree relative with T2D [15]. Meta-analyzes published in the last 50 years show that women with GDM have a higher risk of developing T2D (RR 7.4) than women with normal glucose tolerance during pregnancy. Women with GDM are more likely to develop Metabolic Syndrome, cardiovascular disease, and depression later in life $[16,17]$. Recent analyzes have shown that GDM increases the risk of developing T2D and cardiovascular disease (CVD) in both mother and child [14].

The aim of the present study was to analyze the frequency and the role of some risk factors for the development of Hyperglycemia during pregnancy - age, obesity / overweight, family history of diabetes, previous GDM or high blood sugar before pregnancy, and to assess their influence on the Bulgarian population of pregnant women.

\section{Study Design}

The study was as cross-sectional multicenter population-based, conducted from September 25 to November 6, 2019, in ten regions of Bulgaria (Sofia, Samokov, Pirdop, Smolyan, Gotse Delchev, Gabrovo, Troyan, Burgas, Stara Zagora, Pleven) and their adjacent towns and villages or a total of 84 settlements. We turned to 104 endocrinologists and gynecologists for cooperation, who invited more than 630 pregnant women to participate in the screening. Of these, 547 pregnant women $(86.8 \%)$ took part.

\section{Material}

The mean age of the 547 pregnant women screened was 30.49 \pm 5.12 years, Median 30.00 (minimum 18 - maximun 47), distributed by age periods, table 1 .

Table 1. Distribution of 547 pregnant women by age.

\begin{tabular}{lll}
\hline Age (years) & Number & Percentage \\
\hline $18-22$ & 33 & 6 \\
$23-27$ & 115 & 21 \\
$28-32$ & 219 & 40 \\
$33-37$ & 128 & 23.4 \\
$38-42$ & 46 & 8.4 \\
$43-47$ & 6 & 1.2 \\
\hline
\end{tabular}

The examined pregnant women were distributed by trimesters resp. gestational week (g. w.) as follows: first (up to 12 g.w.) - 110 (20.1\%), second (13-24 g.w.) - 276 (50.5\%), third (after 24 g.w.) - 161 (29.4\%). All participants signed informed consent, confirmed by the local Ethics Commission at Sofiamed University Hospital, Sofia University "Saint Kliment Ohridski". It was prepared following the ethical 
standards according to the Helsinki Declaration-1964 and later additions [18]. Each pregnant woman filled in a Questionnaire with the assistance of a specially designated medical person from the "face to face" team in order to correctly collect data on pregnancy history, past health history, family history for diabetes, GDM in the past, hypertension, thyroid and chronic kidney disease, current and past medication, smoking. All participants were Caucasian, with no evidence of liver, kidney disease or malabsorption.

\section{Methods}

\subsection{The Weight and Height Resp. BMI - Before and During Pregnancy; Arterial Pressure}

After completing the personal Questionnaire, the current weight and height of each pregnant woman were measured and filled in the Questionnaire, together with the weight before pregnancy. The body mass index (BMI $-\mathrm{kg} / \mathrm{m}^{2}$ ) before pregnancy and the current one at the time of the study were calculated. Average weight before pregnancy was $63.68 \pm 14.36$ $\mathrm{kg}$, Median 60.00 (minimum 40 - maximun 166), and weight at the time of screening was $70.59 \pm 14.50 \mathrm{~kg}$, Median 68.00 (minimum 41 - maximun 166). BMI before pregnancy was $23.36 \pm 5.05 \mathrm{~kg} / \mathrm{m}^{2}$, Median 22.06 (minimum 15.24 - maximun 63.25 ), and BMI at the time of screening was $25.91 \pm 5.11 \mathrm{~kg} /$ $\mathrm{m}^{2}$, Median 25.25 (minimum 16.51 - maximun 63.25).

In addition, arterial blood pressure was measured in a sitting position after a 5-minute rest.

\subsection{Laboratory Analysis}

A two-hour, $75 \mathrm{~g}$ oral glucose tolerance test (OGTT) was performed. Venous blood was drawn at 0,60 and 120 minutes in plasma tubes, containing Na2EDTA and $\mathrm{NaF}$, as an inhibitor of glycolysis for stability of glucose in the samples. The samples were transported to the laboratory after centrifugation. All of the samples were analyzed in one Central laboratory on the day of the blood sampling. Glucose was quantitatively determined using enzymatic reference method with hexokinase (Roche reagent) on Cobas e501 analyzer. The results were in mmol / $\mathrm{L}$ established precision using human samples and controls: Intra assay: Level $1(\mathrm{n}=6)$ $\mathrm{CV}=1.12 \%$; Level $2(\mathrm{n}=6) \mathrm{CV}=0.42 \%$; Inter assay: Level 1 $(\mathrm{n}=30) \mathrm{CV}=1.25 \%$; Level $2(\mathrm{n}=30) \mathrm{CV}=1.58 \%$. Two levels Intra laboratory quality control on a daily basis was performed.
The Laboratory participates in two EQA systems Bulgarian EQAS and INSTAND and has certificates for this parameter.

\subsection{The Statistical Analysis}

The statistical analysis was performed using standard SPSS 13.0 for Windows: descriptive statistics (mean, medians, standard deviation), correlation analysis and analysis of variance (ANOVA, post-hoc test - with Bonferroni alpha correction), using parametrical and non-parametrical methods, including - Chi-Square Test, Fisher's Exact Test, Kolmogorov-Smirnov, Shapiro-Wilk Tests, Levene's Test for Equality of Variances, Student's t-test, Kruskal-Wallis test and Mann -Whitney test. All quantitative variables were presented as mean with standard deviation, median or percentage (unless specified otherwise), p-values below 0.05 were accepted as statistically significant.

\section{Results}

The analysis of the results of the conducted classic OGTT, according to the criteria of WHO (2019), FIGO (2015), NICE (2015) for screening during pregnancy, showed that $79 / 547$ (14.4\%) of the screened pregnant women have Hyperglycemia in fasting state or during the test, and in the remaining 468/547 $(85.6 \%)$ pregnant women Normoglycemia was found. With hyperglycemia up to 24 g.w. turned out to be 29/386 pregnant women $(7.5 \%)$ - group 1 , and after 24 g.w. four times more $50 / 161$ (31\%) - group 2, $\mathrm{P}<0.01$.

\subsection{Advanced Maternal Age}

The mean age of the examined pregnant women was $30.49 \pm 5.12 \mathrm{y}$, median 30.00 (min-18, max-47). The age of the group of pregnant women with Hyperglycemia (n-79) versus the others with Normoglycemia (n-468) was significantly higher - 32.18 \pm 5.26 years v.s. 30.21 \pm 5.05 years (Mean Rank $320,84$ v.s. 266,09 - Mann-Whitney Test, $\mathrm{P}<0.005)$ and the same significance is found when comparing group 2 of 50 pregnant women (with Hyperglycemia after 24 g.w.) and the remaining 497 pregnant women $-32.72 \pm 5.25$ v.s. $30.27 \pm 5.06$ (Mean Rank 338,73 v.s. 267,49 - Mann-Whitney Test, $\mathrm{P}<0.002$ ). Regarding group 1 of 29 pregnant women (with Hyperglycemia up to 24 g.w.) no significant difference was found compared with the age of the remaining 518 pregnant women $-31.24 \pm 5.23$ y v.s. $30.45 \pm 5.12$ y (NS), Table 2 .

Table 2. Comparison of age at different glycemic levels in total, before and after 24 g.w.

\begin{tabular}{l|ll}
\hline Level of glycemia & Mean \pm SD & Median (min-max) \\
\hline Normoglycemia (total), n-468 & $30.21 \pm 5.05^{*}$ & $30.00(18-47)$ \\
Hyperglycemia (total), n-79 & $32.18 \pm 5,26^{*}$ & $32.00(20-45)$ \\
Hyperglycemia up to 24 g.w, n-29 & $31.24 \pm 5.23$ & $31.00(23-44)$ \\
Remaining pregnant women from the group up to 24 g.w, n-518 & $30.45 \pm 5.12$ & $30.00(18-47)$ \\
Hyperglycemia after 24 g.w, n-50 & $32.72 \pm 5.25^{* *}$ & $32.50(20-45)$ \\
Remaining pregnant women from the group after 24 g.w, n-497 & $30.27 \pm 5.06^{* *}$ & $30.00(18-47)$ \\
Total, n-547 & $30.49 \pm 5.12$ & $30.00(18-47)$ \\
\hline
\end{tabular}

$* \mathrm{P}<0.005, * * \mathrm{P}<0.002$ 


\subsection{BMI Before Pregnancy}

BMI before pregnancy is a major factor in the manifestation of glycemic disorders in the whole population, as well as in pregnant women. With regard to BMI before pregnancy, this relationship proved to be significant when comparing the mean ranks for Hyperglycemia - total (Mean Rank 260.42 against 354.44 - Mann-Whitney Test, $\mathrm{P}<0.001$ ), for Hyperglycemia up to 24 g.w. (Mean Rank 266.44 against 409.02 - Mann-Whitney Test, $\mathrm{P}<0.001$ ) and for Hyperglycemia after 24 g.w. (Mean Rank 269.09 against 322.78 - Mann-Whitney Test, $\mathrm{P}<0.02$ ) when compared with the respective groups in the three mentioned periods, Table 3. Therefore, women with a higher BMI before pregnancy are significantly at risk of developing glucose tolerance disorders during pregnancy.

Table 3. BMI before pregnancy in women with Hyperglycemia - total, before and after $24 \mathrm{~g}$.w. compared to the respective groups for the indicated periods of pregnancy.

\begin{tabular}{lll}
\hline BMI before pregnancy at different levels of glycemia & Mean \pm SD & Median (min-max) \\
\hline Normoglycemia (total), n-468 & $22.89 \pm 4.57^{*}$ & $22(15-50)$ \\
Hyperglycemia (total), n-79 & $26.10 \pm 6.65^{*}$ & $25(18-63)$ \\
Hyperglycemia up to 24 g.w, n-29 & $28.38 \pm 8.12^{*}$ & $27(20-63)$ \\
Remaining pregnant women from the group up to 24 g.w, n-518 & $23.08 \pm 4.67 *$ & $22(15-50)$ \\
Hyperglycemia after 24 g.w, n-50 & $24.77 \pm 5.29 * *$ & $24(18-41)$ \\
Remaining pregnant women from the group after 24 g.w, n-497 & $23.21 \pm 5.01 * *$ & $21(15-63)$ \\
Total, n-547 & $23.36 \pm 5.05$ & $22(15-63)$ \\
\hline
\end{tabular}

$* \mathrm{P}<0.0001$ and $* * \mathrm{P}<0.02-$ Hyperglycemis versus the remaining pregnant women from the respective groups

\subsection{BMI During Pregnancy}

BMI during pregnancy plays significant role when comparing the mean ranks for Hyperglycemia - total (Mean Rank 257.24 versus 373.28 - Mann-Whitney Test, $\mathrm{P}<0.0001)$, for Hyperglycemia up to 24 g.w. (Mean Rank 268.34 versus 375.14 - Mann-Whitney Test, $\quad \mathrm{P}<0.0001$ ) and for
Hyperglycemia after 24 g.w. (Mean Rank 264.12 versus 372.20 - Mann-Whitney Test, $\mathrm{P}<0.0001$ ), with the respective groups in the three mentioned periods, Table 4 . Therefore, women with higher BMI during pregnancy are also significantly threatened by the development of glucose tolerance disorders in this period of their lives.

Table 4. BMI during pregnancy in women with Hyperglycemia - total, up to and after 24 g.w. compared to the respective groups for the indicated periods of pregnancy.

\begin{tabular}{llll}
\hline BMI during pregnancy at different levels of glycemia & Mean \pm SD & Median (min-max) & $24.6(16-49)$ \\
\hline Normoglycemia (total), n-468 & $25.35 \pm 4.66^{*}$ & $29.1(20-63)$ \\
Hyperglycemia (total), n-79 & $29.94 * 8.17^{*}$ & $28.9(20-63)$ \\
Hyperglycemia up to 24 g.w. n-29 & $25.69 \pm 4.80^{*}$ & $25(16-49)$ \\
Remaining pregnant women from the group up to 24 g.w, n-518 & $28.86 \pm 4.96^{*}$ & $27.8(20-43)$ \\
Hyperglycemia after 24 g.w. n-50 & $25.62 \pm 5.03 *$ & $24.9(16-63)$ \\
Remaining pregnant women from the group after 24 g.w. n-497 & $25.92 \pm 5.11$ & $25.2(16-63)$ \\
Total, n-547 & & \\
\hline
\end{tabular}

$* \mathrm{P}<0.0001-$ Hwperglycemia v.s. remaining pregnant women from the respective group

\subsection{Family Burden with Diabetes}

It was found that $15.7 \%$ of all pregnant women (n-86/547) had a family history of diabetes. Family history of diabetes occurred in $29.1 \%(23 / 79)$ of pregnant women with Hyperglycemia, versus $13.5 \%$ (63/468) of pregnant women with Normoglycemia, $\mathrm{P}<0.001$.

\subsubsection{Family Burden with Diabetes (up to 24 g.w.)}

In the subgroup of 386 pregnant women up to 24 g.w. (29 with Hyperglycemia and 357 with Normoglycemia) family history of Diabetes was present in a total of $18.6 \%(72 / 386)$. The frequency of family burden with Diabetes was significantly higher among 29 pregnant women with Hyperglycemia up to 24 g.w. 31\% (9/29) compared with women with Normoglycemia in this subgroup - $17.6 \%$ (63/357), $\mathrm{P}<0.001$.

\subsubsection{Family Burden with Diabetes (After 24 g.w.)}

In the subgroup of 161 pregnant women after 24 g.w. (50 with Hyperglycemia and 111 with Normoglycemia) a family history of diabetes occurred in $8.7 \%(14 / 161)$. It turned out that a family history of diabetes is present in $28 \%(14 / 50)$ of the pregnant women with Hyperglycemia after 24 g.w. and was absent in the remaining 111 pregnant women with Normoglycemia during this period of pregnancy.

\subsection{Previous Gestational Diabetes}

Previous Gestational diabetes was present in 6 of 547 pregnant women or $1.1 \%$. There was a significant difference between the incidence of Hyperglycemia in pregnant women with previous GDM compared with those who did not have $3 / 79$ (3.8\%) versus 3/468 (0.6\%), Fisher's Exact Test, $\mathrm{P}<0.04$. Hyperglycemia up to 24 g.w. was also significantly more common in women with previous GDM - 2/29 (6.9\%) compared to those who did not have 4/518 (0.8\%), Fisher's Exact Test, $\mathrm{P}<0.036$. Regarding existing Hyperglycemia after 24 g.w. no significant association was found in women with or 
without GDM (2\% vs. $1 \%$, NS).

\subsection{High Blood Sugar Before Pregnancy}

In $1.46 \%$ of pregnant women $(8 / 547)$ there were data for accidental elevated blood sugar before the current pregnancy. In $50 \%$ of them (4/8) in the current screening Hyperglycemia was found against $13.9 \%(75 / 539)$ in women without elevated blood sugar before the current pregnancy, $\mathrm{P}<0.018$. Hyperglycemia up to 24 g.w. was found in $25 \%(2 / 8)$ of women with elevated blood sugar before the current pregnancy against $5 \%(27 / 539)$ of women without previous elevated blood sugar, $\mathrm{P}<0.062$. Hyperglycemia after 24 g.w. was detected in $25 \%(2 / 8)$ of women with previous elevated blood sugar versus $8.9 \%(48 / 539)$ in women without this previous disorder, NS. Elevated blood sugar before pregnancy is a significant risk factor for the occurrence of Hyperglycemia during pregnancy. It is extremely important for the development of Hyperglycemia up to 24 g.w. and has no significant value for the development of the Hyperglycemia after 24 g.w.

There was a significant correlation between elevated blood sugar before the current pregnancy and the incidence of Hyperglycemia in the whole group of 547 pregnant women $(\mathrm{P}<0.004)$, as well as in the subgroup of pregnant women up to 24 g.w. $(\mathrm{P}<0.01)$. No such relationship was found in the subgroup of pregnant women after 24 g.w. (NS).

\section{Discussion}

Before discussing the frequency and characteristics of the types of Glucose tolerance disorders among Bulgarian pregnant women, several facts from the two population screenings for diabetes (2006 and 2012) in Bulgaria should be presented [19]. It should be taken into account that there has been a considerable economic emigration involving 20-40-year-old population since 1990, and as a result, birth rate has been declining, and the population has been aging. At the same time, the unemployment rate has been high and the percentage of the population with low-income has been increasing [20]. A total change in diabetes prevalence (known and unknown) for the studied period from $7.9 \%(190 / 2396)$ in 2006 to $9.55 \%$ $(194 / 2033)$ in 2012 was found, $\mathrm{P}<0.06$. The relative increase was by $20.88 \%$ or $3.5 \%$ per year. Increase in diabetes prevalence was found in both genders, but more markedly in the males, in whom it was marginally significant (by $2.3 \%$ or a relative increase of $25 \%-9.2 \%$ in 2006 vs. $11.5 \%$ in 2012 , $\mathrm{P}=0.06$ ). In the females, the increase was minimal, from $6.9 \%$ in 2006 to $7.8 \%$ in 2012 , a relative increase of $13 \%$ (NS). An increase was observed also in the obesity prevalence in the general population from $26.7 \%(640 / 2392)$ in 2006 to $32.7 \%$ $(661 / 2021)$ in $2012, \mathrm{P}=0.02$, a relative increase of $22.4 \%$ [21]. It was also found that in young women (20-44 years) the incidence of diabetes was $12.9 \%(8 / 62)$ [22]. According to the latest IDF data from 2019, the incidence of diabetes in Bulgaria has increased and Diagnosed diabetes was 8.3\%, and Undiagnosed $-2.7 \%$ or total diabetes has reached $11.0 \%$ [1].

The incidence of GDM also increases with age and it usually reflects the incidence of $\mathrm{T} 2 \mathrm{D}$ in a given population [23]. Growing maternal age, the epidemic of obesity and diabetes, as well as reduced physical activity are reasons for the increase in the frequency of GDM, as well as Diabetes in Pregnancy - DIP (each of them meets specific criteria for oGTT) [24, 25]. Hyperglycemia first detected at any time during pregnancy should be classified either as DIP or GDM [26].

The 2015 NICE Recommendations, as well as those of other international organizations, precisely list the risk factors for GDM - BMI $\geq 30 \mathrm{~kg} / \mathrm{m}^{2}$, previous macrosomic baby weighing $4.5 \mathrm{~kg}$ or above, previous Gestational diabetes, family history of diabetes (first-degree relative with diabetes) [7]. Each of these risk factors is directly or indirectly related to $\beta$-cell dysfunction and / or decreased peripheral insulin sensitivity. For example, overweight and obesity are inextricably linked to systemic, excessive calorie intake, which permanently increases insulin production by $\beta$-cells and this adversely affects insulin signaling pathways [14]. As a result of prolonged increased insulin production in response to excessive caloric intake, $\beta$-cell dysfunction develops [27]. In turn, hyperinsulinism based on $\beta$-cell dysfunction on the one hand exacerbates insulin resistance by about $54 \%$ according to Catalano, P.M, 2014 [28] and on the other hand realizes glucotoxicity on $\beta$-cells, impairing their performance [29]. It should be noted that insulin resistance during pregnancy develops physiologically in connection with the hormonal changes that occur during this period, but in obesity, insulin resistance becomes too high and exceeds physiological limits. Thus, obesity / overweight leads to such disorders as glucose intolerance and GDM.

This fact is also present in our material, where the group of pregnant women with Hyperglycemia has a significantly higher BMI before pregnancy compared to the group of pregnant women with Normoglycemia - $26.10 \pm 6.65 \mathrm{~kg} / \mathrm{m}^{2}$ against $22.89 \pm 4.57 \mathrm{~kg} / \mathrm{m}^{2}, \mathrm{P}<0.0001$. The same was found when comparing BMI during pregnancy between the two groups - 29.26 $\pm 6.27 \mathrm{~kg} / \mathrm{m}^{2}$ against $25.35 \pm 4.66 \mathrm{~kg} / \mathrm{m}^{2}$, $\mathrm{P}<0.0001$. If we compare the two periods before and after 24 g.w. the same significant difference was present between the two groups with Hyperglycemia resp. with Normoglycemia both before and during pregnancy.

All this illustrates the permanence of the disorder and its very deep genesis. We may conclude, that obesity / overweight ranks first as a risk factor for diabetes among the population and for GDM in particular, according to all international guidelines.

Previous GDM as well as elevated blood sugar before pregnancy are the other main risk factors for GDM. In fact for women who have already shown slight deviations in glucose tolerance, their new pregnancy has additional strong impact on $\beta$-cells and on the process by which insulin action is realized in peripheral tissues. All this leads to the development of glucose intolerance and GDM.

In the 547 pregnant women screened, in $1.1 \%$ we found a previous GDM and in $1.46 \%$ - elevated blood sugar before the current pregnancy. A significant difference in the frequency 
was found for both disorders between the groups with Hyperglycemia resp. with Normoglycemia - 3.8\% (3/79) against $0.6 \%$ (3/468), $\mathrm{P}<0.04$ for GDM and resp. 50\% (4/8) versus $13.9 \%(75 / 539), \mathrm{P}<0.018$ in the group with previous elevated blood sugar. Interestingly, the same significant difference was found for both disturbances in the period up to 24 g.w. $(\mathrm{P}<0.036$ resp $\mathrm{P}<0.062)$, while for the period after 24 g.w. it was not found. Therefore, the presence of potential disturbances in glucose metabolism very early in pregnancy inreases and dysglycemia manifests itself.

In our population screening from 2012 in 2032 persons (953 men and 1079 women, 20-80 years) family history of diabetes was present in $19.4 \%$ [30]. In the current screening of 547 Bulgarian pregnant women, family history of diabetes was $15.7 \%(86 / 547)$. We found twice as often a family history in pregnant women with Hyperglycemia 29.1\% (23/79) versus $13.5 \%(63 / 468)$ in those with Normoglycemia, $\mathrm{P}<0.001$. It is impressive that during the period up to 24 g.w. the frequency of the family burden with diabetes is $18.6 \%(72 / 386)$, and during the period after 24 g.w. is significantly less $-8.7 \%$ (14/161), $\mathrm{P}<0.001$. Therefore, familial burden with diabetes is a very strong risk factor for the development of hyperglycemia even up to 24 g.w.

A family history of diabetes was registered more often among pregnant women up to 24 g.w. with Hyperglycemia compared to women with Normoglycemia - 31\% (9/29) vs. $17.6 \%$ (63/357), $\mathrm{P}<0.001$. For the period after 24 g.w. only 14 pregnant women with a family history of Diabetes remained and all of them were in the group with Hyperglycemia - $28 \%$ $(14 / 50)$, while the other 111 pregnant women had Normoglycemia and no family history of diabetes. Therefore, the family burden of diabetes is a very strong factor, which up to 24 g.w. exerts its effect on the glycemic control system.

It is a well known fact that the incidence of diabetes (known and unknown) increases with age. In the previous Bulgarian population screening from 2012 which included 2032 people, it was found that $9.6 \%$ of the population had diabetes (known $7.1 \%$ and unknown $-2.5 \%$ ). By age categories, diabetics were distributed as follows: young age (20-44 years) - $9.8 \%$, middle age (45-59 years) - $34.5 \%$ and in the third age ( $\geq 60$ years) $55.7 \%$ [30]. In the subgroup of young women (20-45 years) the incidence of diabetes was estimated at $12.9 \%(8 / 62)$ [22].

In our pregnant cohort, the first thing that could be noted is the change in the age profile of the Bulgarian pregnant women in the last few years. Compared to the 2015, Euro-Peristat Project population, five years later, in 2019, the percentage of pregnancies below the age of 20 has decreased in Bulgaria (from $10.2 \%$ to $2.7 \%$, respectively), as well as in the range between 20 and 24 years (from 21.7 to $7.9 \%$, respectively). At the same time there was an increase in the percentage of pregnant women between 25 and 34 years (from $54.5 \%$ to $68.5 \%$ ) and $35+$ years (from $13.6 \%$ to $20.8 \%$, respectively) [31].

In the present material, the mean age of 547 pregnant women was $30.49 \pm 5.12$ years, divided into age periods. As can be seen from Table 1 , over $73 \%$ of the studied pregnant women, were in the age range from 28 to 47 years. Pregnant women with Hyperglycemia (n-79) were significantly older compared to the group with Normoglycemia (n-468) $32.18 \pm 5.26$ years v.s. $30.21 \pm 5.05$ years, $\mathrm{P}<0.005$.

When comparing the groups after 24 g.w. with Hyperglycemia (n-50) and pregnant women with Normoglycemia $(n-497)$ the difference in their age is again significant $-32.72 \pm 5.25$ y v.s. $30.27 \pm 5.06$ y, $\mathrm{P}<0.002$. Age does not show a significant difference only between the two groups in the period up to 24 g.w. Thus, it can be concluded that the age-diabetes relationship is direct and the tendency for increasing the maternal age of women in the country predetermines the expectation for a rise in the incidence of Diabetes mellitus (GDM or DIP).

\section{Conclusion}

Considering the main significant risk factors for the development of Hyperglycemia during pregnancy, identified in our population in the current screening - advanced maternal age, obesity / overweight, family history of Diabetes, previous Gestational diabetes or elevated blood sugar before pregnancy, verbal screening would be very helpful and should be an indicator for the need to screen each pregnant woman with any of these risk factors.

This will promptly diagnose the most common disorder during pregnancy - Hyperglycemia, and will have very high medical and social significance. It should be emphasized that some of the risk factors are subject to modification. One such factor is age of pregnancy, which is progressively increasing due to many social, financial and personal reasons. Another factor is obesity/overweight which is very common among the whole population. These are both major risk factors for diabetes (Gestational Diabetes mellitus - GDM or Diabetes in Pregnancy - DIP). They can be modified and are the main reason for testing pregnant women for diabetes when pregnancy is first diagnosed.

\section{Conflicts of Interests}

All the authors do not have any possible conflicts of interest.

\section{Acknowledgements}

The authors thank the endocrinologists and obstetrician/gynecologists who provided local support: V. Jotova (Troyan), S. Dimitrova (Gabrovo), Anchev (Gabrovo), Mitev (Gabrovo), A. Popov (Goce Delchev), A. Andreev (Samokov), E. Apostolova (Smolyan), M. Hubshev (Smolyan), S. Dimitrov (Pirdop), R. Naumovska (Pleven), M. Manuelyan (Burgas), K. Kirovakov (Burgas), T. Gabrovska (Burgas), K. Venkova (Sofia), M. Angelova (Stara Zagora), R. Velev (Sofia), I. Sigridov (Sofia), B. Bogoslovova (Sofia)... total 104 specialists in the country. Technical support: T. Kornilova (Sofia), Z. Metodieva (Sofia), J. Georgieva (Sofia), H. Atanasova (Burgas), I. Ilieva (Stara Zagora). This work was supported by grants from the Bulgarian Society of Endocrinology (Project Number 7/2019) as part of the National Epidemiological Program for Pregnant Women in 


\section{References}

[1] International Diabetes Federation. (2019). IDF Diabetes Atlas, $9^{\text {th }}$ edn. Brussels, Belgium: International Diabetes Federation.

[2] Diabetes mellitus. Report of a WHO Expert Committee. Geneva: World Health Organization; 1965.

[3] World Health Organization (1999): Definition, diagnosis and classification of diabetes mellitus and its complications. Part 1: Diagnosis and Classification of Diabetes Mellitus. Geneva: World Health Organization.

[4] Diagnostic criteria and classification of hyperglycemia first detected in pregnancy: a World Health Organization Guideline. (2014). Diab Res Clin Pract 103: 341-63.

[5] Hod M, Kapur A, Sacks D. A, Hadar E, Agarwal M, Di Renzo G. C, Roura L. C, McIntyre H. D, Morris J. L, Divakar H. The International Federation of Gynecology and Obstetrics - FIGO. (2015). Initiative on gestational diabetes mellitus: A pragmatic guide for diagnosis, management, and care. International Journal of Gynecology and Obstetrics 131, S3, S173-S211.

[6] World Health Organization. (2019). Classification of Diabetes mellitus 2019. ISBN 978-92-4-151570-2.

[7] NICE. (2015). Diabetes in pregnancy: management from preconception to the postnatal period. https://www.nice.org.uk/terms-and conditions\#notice-ofrights.

[8] International Diabetes Federation. (2013). IDF Atlas. Sixth Edition. Brussels, Belgium: International Diabetes Federation.

[9] Guariguata L, Linnenkamp U, Beagley J, Whiting DR, Cho NH (2014). Global estimates of the prevalence of hyperglycemia in pregnancy. Diabetes Res Clin Pract 103 (2): 176-185. https://doi. org/10.1016/j.diabres.2013.11.003.

[10] Melchior H, Kurch-Bek D, Mund M. (2017). The prevalence of gestational diabetes. Dtsch Arztebl Int 114: 412-418. https://doi. org/10.3238/arztebl.2017.0412.

[11] Borissova A-M, Trifonova B, Dakovska L, Michaylova E, Vukov M. (2021). Cross-sectional study of glucose disturbances of pregnant women in Bulgaria. Clinical Medicine Research 10 (1): 9-15.

[12] McCance D, Maresh M, Sacks DA. (2017). A practical manual of diabetes in pregnancy. John Wiley \& Sons. doi: $10.1002 / 9781119043805$.

[13] Ferrara A. (2007). Increasing prevalence of gestational diabetes mellitus: a public health perspective. Diabetes Care 30 (Suppl 2): S141-146. doi: 10.2337/dc07-s206.

[14] Plows J. F, Stanley J. L, Baker P. N, Reynolds C. M, Vickers M H. (2018). The Pathophysiology of Gestational Diabetes Mellitus. Int. J. Mol. Sci. 19, 3342; doi: 10.3390/ijms19113342.

[15] Wagner R, Thorand B, Osterhoff M. A, Müller G, Böhm A, Meisinger C, Kowall B, Rathmann W, Kronenberg F, Staiger H, Stefan N, Roden M, Schwarz P. E, Pfeiffer A. F, Häring H-U, Fritsche A. (2013). Family history of diabetes is associated with higher risk for prediabetes: a multicentre analysis from the German Center for Diabetes Research. Diabetologia 56: 2176-
[16] Hakkarainen H, Huopio H, Cederberg H, Pääkkönen $M$, Voutilainen R, Heinonen S. (2016). The risk of metabolic syndrome in women with previous GDM in a long-term follow-up. Gynecol Endocrinol 32 (11): 920-925. https://doi.org/10.1080/09513590. 2016.1198764.

[17] Kramer CK, Campbell S, Retnakaran R. (2019). Gestational diabetes and the risk of cardiovascular disease in women: a systematic review and meta-analysis. Diabetologia 62 (6): 905-914. https://doi. org/10.1007/s00125-019-4840-2.

[18] World Medical Association. (2013). "Declaration of Helsinki: Ethical Principles for Medical Research Involving Human Subjects". JAMA. 310 (20): 2191-2194. doi: 10.1001/jama.2013.28105.

[19] Borissova A-M, Shinkov A, Vlahov J, Dakovska L, Blajeva E, Todorov T. (2012). Prevalence of diabetes mellitus and prediabetes in Bulgaria today. Endokrinologya 17: 172-182.

[20] Poverty Reduction and Economic Management Unit. (2013). Mitigating the Economic Impact of an Aging Population Options for Bulgaria. Washington, DC: The World Bank.

[21] Borissova A-M, Shinkov A, Kovatcheva R, Vlahov J, Dakovska L, Todorov T. (2015). Changes in the Prevalence of Diabetes Mellitus in Bulgaria (2006-2012). Clinical Medicine Insights: Endocrinology and Diabetes 8, 41-45.

[22] Borissova A-M, Shinkov A, Vlahov J, Dakovska L, TodorovT. (2016). Higher Prevalence of Diabetes Mellitus and Impaired Glucose Tolerance Among the Rural Population in Bulgaria. J Endocrinol Metab Vol 6, Number 2, 52-58.

[23] King H. (1998). Epidemiology of glucose intolerance and gestational diabetes in women of childbearing age. Diabetes Care 21 (Suppl. 2): B9-B13.

[24] Births: Final Data for 2002. Atlanta, GA, Centers for Disease Control and Prevention, December 2003, DHHS publ. no. PHS 2004-1120.

[25] Behavioral Risk Factor Surveillance System: Accessed 25 October 2005 at http://apps.nccd.cdc.gov/brfss/trends.

[26] World Health Organization. (2014). Obesity and overweight. Fact sheet $\quad \mathrm{N}^{\circ} 311$. http:// www.who.int/mediacentre/factsheets/fs311/en/. Updated January 2015. Accessed March 202014.

[27] Weir G. C, Laybutt D. R, Kaneto H, Bonner-Weir S, Sharma A. (2001). Beta-cell adaptation and decompensation during the progression of diabetes. Diabetes 50 (Suppl. 1), S154-159.

[28] Catalano, P. M. (2014). Trying to understand gestational diabetes. Diabet Med 31, 273-281.

[29] Ashcroft F. M, Rohm M, Clark A, Brereton M. F. (2017). Is Type 2 Diabetes a Glycogen Storage Disease of Pancreatic $\beta$-Cells? Cell 26, 17-23.

[30] Borissova A-M, Shinkov A, Vlahov J, Dakovska L, Metalova T. Screening for endocrine diseases in the Bulgarian population ( $\geq 20-80$ years), preliminary data. $15^{\text {th }}$ National Symposium on Endocrinology, 19-21 April 2012, Plovdiv, Bulgaria.

[31] Euro-Peristat Project. (2018). European Perinatal Health Report. Core indicators of the health and care of pregnant women and babies in Europe in 2015. www.europeristat.com. 